\title{
A Lynden-Bell integral estimator for the tail index of right-truncated data with a random threshold
}

\author{
Nawel Haouas, Abdelhakim Necir ${ }^{1}$, Djamel Meraghni, Brahim Brahimi \\ Laboratory of Applied Mathematics, Mohamed Khider University, Biskra, Algeria
}

\begin{abstract}
By means of a Lynden-Bell integral with deterministic threshold, Worms and Worms [A Lynden-Bell integral estimator for extremes of randomly truncated data. Statist. Probab. Lett. 2016; 109: 106-117] recently introduced an asymptotically normal estimator of the tail index for randomly right-truncated Pareto-type data. In this context, we consider the random threshold case to derive a Hill-type estimator and establish its consistency and asymptotic normality. A simulation study is carried out to evaluate the finite sample behavior of the proposed estimator.
\end{abstract}

Keywords: Extreme value index; Heavy-tails; Lynden-Bell estimator; Random truncation. AMS 2010 Subject Classification: 60F17, 62G30, 62G32, 62P05.

\section{Introduction}

Let $\left(\mathbf{X}_{i}, \mathbf{Y}_{i}\right), 1 \leq i \leq N$ be a sample of size $N \geq 1$ from a couple $(\mathbf{X}, \mathbf{Y})$ of independent random variables (rv's) defined over some probability space $(\Omega, \mathcal{A}, \mathbf{P})$, with continuous marginal distribution functions (df's) $\mathbf{F}$ and $\mathbf{G}$ respectively. Suppose that $\mathbf{X}$ is truncated to the right by $\mathbf{Y}$, in the sense that $\mathbf{X}_{i}$ is only observed when $\mathbf{X}_{i} \leq \mathbf{Y}_{i}$. We assume that both survival functions $\overline{\mathbf{F}}:=1-\mathbf{F}$ and $\overline{\mathbf{G}}:=1-\mathbf{G}$ are regularly varying at infinity with respective negative indices $-1 / \gamma_{1}$ and $-1 / \gamma_{2}$. That is, for any $x>0$,

$$
\lim _{z \rightarrow \infty} \frac{\overline{\mathbf{F}}(x z)}{\overline{\mathbf{F}}(z)}=x^{-1 / \gamma_{1}} \text { and } \lim _{z \rightarrow \infty} \frac{\overline{\mathbf{G}}(x z)}{\overline{\mathbf{G}}(z)}=x^{-1 / \gamma_{2}} .
$$

It is well known that, in extreme value analysis, weak approximations are achieved in the second-order framework (see, e.g., de Haan and Ferreira, 2006, page 48). Thus, it seems

\footnotetext{
${ }^{1}$ Corresponding author: necirabdelhakim@yahoo.fr

E-mail addresses:

nawel.haouas@yahoo.com (N. Haouas)

djmeraghni@yahoo.com (D. Meraghni)

brah.brahim@gmail.com (B. Brahimi)
} 
quite natural to suppose that $\mathbf{F}$ and $\mathbf{G}$ satisfy the second-order condition of regular variation, which we express in terms of the tail quantile functions pertaining to both df's. That is, we assume that for $x>0$, we have

$$
\lim _{t \rightarrow \infty} \frac{\mathbb{U}_{\mathbf{F}}(t x) / \mathbb{U}_{\mathbf{F}}(t)-x^{\gamma_{1}}}{\mathbf{A}_{\mathbf{F}}(t)}=x^{\gamma_{1}} \frac{x^{\tau_{1}}-1}{\tau_{1}},
$$

and

$$
\lim _{t \rightarrow \infty} \frac{\mathbb{U}_{\mathbf{G}}(t x) / \mathbb{U}_{\mathbf{G}}(t)-x^{\gamma_{2}}}{\mathbf{A}_{\mathbf{G}}(t)}=x^{\gamma_{2}} \frac{x^{\tau_{2}}-1}{\tau_{2}},
$$

where $\tau_{1}, \tau_{2}<0$ are the second-order parameters and $\mathbf{A}_{\mathbf{F}}, \mathbf{A}_{\mathbf{G}}$ are functions tending to zero and not changing signs near infinity with regularly varying absolute values at infinity with indices $\tau_{1}, \tau_{2}$ respectively. For any df $K$, the function $\mathbb{U}_{K}(t):=K^{\leftarrow}(1-1 / t), t>1$, stands for the tail quantile function, with $K^{\leftarrow}(u):=\inf \{v: K(v) \geq u\}, 0<u<1$, denoting the generalized inverse of $K$. From Lemma 3 in Hua and Joe (2011), the second-order conditions (1.2) and (1.3) imply that there exist constants $d_{1}, d_{2}>0$, such that

$$
\overline{\mathbf{F}}(x)=d_{1} y^{-1 / \gamma_{1}} \ell_{1}(x) \text { and } \overline{\mathbf{G}}(x)=d_{2} y^{-1 / \gamma_{2}} \ell_{2}(x), x>0,
$$

where $\lim _{x \rightarrow \infty} \ell_{i}(x)=1$ and $\left|1-\ell_{i}\right|$ is regularly varying at infinity with tail index $\tau_{i} \gamma$, $i=1,2$. This ccondition is fullfilled by many commonly used models such as Burr, Fréchet, Generalized Pareto, absolute Student, log-gamma distributions, to name but a few. Also known as heavy-tailed, Pareto-type or Pareto-like distributions, these models take a prominent role in extreme value theory and have important practical applications as they are used rather systematically in certain branches of non-life insurance, as well as in finance, telecommunications, hydrology, etc... (see, e.g., Resnick, 2006).

Let us now denote $\left(X_{i}, Y_{i}\right), i=1, \ldots, n$ to be the observed data, as copies of a couple of rv's $(X, Y)$, corresponding to the truncated sample $\left(\mathbf{X}_{i}, \mathbf{Y}_{i}\right), i=1, \ldots, N$, where $n=n_{N}$ is a sequence of discrete rv's which, in virtue of the weak law of large numbers, satisfies $n_{N} / N \stackrel{\mathbf{P}}{\rightarrow} p:=\mathbf{P}(\mathbf{X} \leq \mathbf{Y})$, as $N \rightarrow \infty$. We denote the joint df of $X$ and $Y$ by $H(x, y):=\mathbf{P}(X \leq x, Y \leq y)=\mathbf{P}(\mathbf{X} \leq \min (x, \mathbf{Y}), \mathbf{Y} \leq y \mid \mathbf{X} \leq \mathbf{Y})$, which is equal to $p^{-1} \int_{0}^{y} \mathbf{F}(\min (x, z)) d \mathbf{G}(z)$. The marginal distributions of the rv's $X$ and $Y$, respectively denoted by $F$ and $G$, are given by $F(x)=p^{-1} \int_{0}^{x} \overline{\mathbf{G}}(z) d \mathbf{F}(z)$ and $G(y)=p^{-1} \int_{0}^{y} \mathbf{F}(z) d \mathbf{G}(z)$. Since $\mathbf{F}$ and $\mathbf{G}$ are heavy-tailed, then their right endpoints are infinite and thus they are equal. Hence, from Woodroofe (1985), we may write $\int_{x}^{\infty} d \mathbf{F}(y) / \mathbf{F}(y)=\int_{x}^{\infty} d F(y) / C(y)$, where $C(z):=\mathbf{P}(X \leq z \leq Y)$. Differentiating the previous equation leads to the following crucial equation $C(x) d \mathbf{F}(x)=\mathbf{F}(x) d F(x)$, whose solution is defined by $\mathbf{F}(x)=$ 
$\exp \left\{-\int_{x}^{\infty} d F(z) / C(z)\right\}$. This leads to Woodroofe's nonparametric estimator (Woodroofe, 1985) of df $\mathbf{F}$, given by

$$
\mathbf{F}_{n}^{(\mathbf{W})}(x):=\prod_{i: X_{i}>x} \exp \left\{-\frac{1}{n C_{n}\left(X_{i}\right)}\right\}
$$

which is derived only by replacing df's $F$ and $C$ by their respective empirical counterparts $F_{n}(x):=n^{-1} \sum_{i=1}^{n} \mathbf{1}\left(X_{i} \leq x\right)$ and $C_{n}(x):=n^{-1} \sum_{i=1}^{n} \mathbf{1}\left(X_{i} \leq x \leq Y_{i}\right)$. There exists a more popular estimator for $\mathbf{F}$, known as Lynden-Bell nonparametric maximum likelihood estimator (Lynden-Bell, 1971), defined by

$$
\mathbf{F}_{n}^{(\mathbf{L B})}(x):=\prod_{i: X_{i}>x}\left(1-\frac{1}{n C_{n}\left(X_{i}\right)}\right)
$$

which will be considered in this paper to derive a new estimator for the tail index of df $\mathbf{F}$. Note that the tail of df $F$ simultaneously depends on $\overline{\mathbf{G}}$ and $\overline{\mathbf{F}}$ while that of $\bar{G}$ only relies on $\overline{\mathbf{G}}$. By using Proposition B.1.10 in de Haan and Ferreira (2006), to the regularly varying functions $\overline{\mathbf{F}}$ and $\overline{\mathbf{G}}$, we show that both $\bar{F}$ and $\bar{G}$ are regularly varying at infinity as well, with respective indices $-1 / \gamma:=-\left(\gamma_{1}+\gamma_{2}\right) /\left(\gamma_{1} \gamma_{2}\right)$ and $-1 / \gamma_{2}$. In view of the definition of $\gamma$, Gardes and Stupfler (2015) derived a consistent estimator, for the extreme value index $\gamma_{1}$, whose asymptotic normality is established in Benchaira et al. (2015), under the tail dependence and the second-order conditions of regular variation. Recently, by considering a Lynden-Bell integration with a deterministic threshold $t_{n}>0$, Worms and Worms (2016) proposed another asymptotically normal estimator for $\gamma_{1}$ as follows:

$$
\widehat{\gamma}_{1}^{(\mathbf{L B})}\left(t_{n}\right):=\frac{1}{n \overline{\mathbf{F}}_{n}^{(\mathbf{L B})}\left(t_{n}\right)} \sum_{i=1}^{n} \mathbf{1}\left(X_{i}>t_{n}\right) \frac{\mathbf{F}_{n}^{(\mathbf{L B})}\left(X_{i}\right)}{C_{n}\left(X_{i}\right)} \log \frac{X_{i}}{t_{n}} .
$$

Likewise, Benchaira et al. (2016a) considered a Woodroofe integration (with a random threshold) to propose a new estimator for the tail index $\gamma_{1}$ given by

$$
\widehat{\gamma}_{1}^{(\mathbf{W})}:=\frac{1}{n \overline{\mathbf{F}}_{n}^{(\mathbf{W})}\left(X_{n-k: n}\right)} \sum_{i=1}^{k} \frac{\mathbf{F}_{n}^{(\mathbf{W})}\left(X_{n-i+1: n}\right)}{C_{n}\left(X_{n-i+1: n}\right)} \log \frac{X_{n-i+1: n}}{X_{n-k: n}},
$$

where, given $n=m=m_{N}, Z_{1: m} \leq \ldots \leq Z_{m: m}$ denote the order statistics pertaining to a sample $Z_{1}, \ldots, Z_{m}$, and $k=k_{n}$ is a (random) sequence of integers such that, given $n=m$, $1<k_{m}<m, k_{m} \rightarrow \infty$ and $k_{m} / m \rightarrow 0$ as $N \rightarrow \infty$. The consistency and asymptotic normality of $\widehat{\gamma}_{1}^{(\mathbf{W})}$ are established in Benchaira et al. (2016a) through a weak approximation 
to Woodroofe's tail process

$$
\mathbf{D}_{n}^{(\mathbf{W})}(x):=\sqrt{k}\left(\frac{\overline{\mathbf{F}}_{n}^{(\mathbf{W})}\left(X_{n-k: n} x\right)}{\overline{\mathbf{F}}_{n}^{(\mathbf{W})}\left(X_{n-k: n}\right)}-x^{-1 / \gamma_{1}}\right), x>0 .
$$

More precisely, the authors showed that, under (1.2) and (1.3) with $\gamma_{1}<\gamma_{2}$, there exist a function $\mathbf{A}_{0}(t) \sim \mathbf{A}_{\mathbf{F}}^{*}(t):=\mathbf{A}_{\mathbf{F}}\left(1 / \overline{\mathbf{F}}\left(\mathbb{U}_{F}(t)\right)\right), t \rightarrow \infty$, and a standard Wiener process $\{\mathbf{W}(s) ; s \geq 0\}$, defined on the probability space $(\Omega, \mathcal{A}, \mathbf{P})$, such that, for $0<\epsilon<1 / 2-\gamma / \gamma_{2}$ and $x_{0}>0$,

$$
\sup _{x \geq x_{0}} x^{(1 / 2-\epsilon) / \gamma-1 / \gamma_{2}}\left|\mathbf{D}_{n}^{(\mathbf{W})}(x)-\mathbf{\Gamma}(x ; \mathbf{W})-x^{-1 / \gamma_{1}} \frac{x^{\tau_{1} / \gamma_{1}}-1}{\gamma_{1} \tau_{1}} \sqrt{k} \mathbf{A}_{0}(n / k)\right|=o_{\mathbf{P}}(1),
$$

as $N \rightarrow \infty$, provided that given $n=m, \sqrt{k_{m}} \mathbf{A}_{0}\left(m / k_{m}\right)=O(1)$, where $\{\Gamma(x ; \mathbf{W}) ; x>0\}$ is a Gaussian process defined by

$$
\begin{aligned}
\boldsymbol{\Gamma}(x ; \mathbf{W}):= & \frac{\gamma}{\gamma_{1}} x^{-1 / \gamma_{1}}\left\{x^{1 / \gamma} \mathbf{W}\left(x^{-1 / \gamma}\right)-\mathbf{W}(1)\right\} \\
& +\frac{\gamma}{\gamma_{1}+\gamma_{2}} x^{-1 / \gamma_{1}} \int_{0}^{1} s^{-\gamma / \gamma_{2}-1}\left\{x^{1 / \gamma} \mathbf{W}\left(x^{-1 / \gamma} s\right)-\mathbf{W}(s)\right\} d s .
\end{aligned}
$$

In view of the previous weak approximation, the authors also proved that if, given $n=m$, $\sqrt{k_{m}} \mathbf{A}_{\mathbf{F}}^{*}\left(m / k_{m}\right) \rightarrow \lambda$, then $\sqrt{k}\left(\widehat{\gamma}_{1}-\gamma_{1}\right) \stackrel{\mathcal{D}}{\rightarrow} \mathcal{N}\left(\frac{\lambda}{1-\tau_{1}}, \sigma^{2}\right)$, as $N \rightarrow \infty$, where $\sigma^{2}:=$ $\gamma^{2}\left(1+\gamma_{1} / \gamma_{2}\right)\left(1+\left(\gamma_{1} / \gamma_{2}\right)^{2}\right) /\left(1-\gamma_{1} / \gamma_{2}\right)^{3}$. Recently, Benchaira et al. (2016b) followed this approach to introduce a kernel estimator to $\gamma_{1}$ which improves the bias of $\widehat{\gamma}_{1}^{(\mathbf{W})}$. In this paper, we are interested in Worm's estimator $\widehat{\gamma}_{1}^{(\mathbf{L B})}\left(t_{n}\right)$, but with a threshold $t_{n}$ that is assumed to be random and equal to $X_{n-k: n}$. This makes the estimator more convenient for numerical implementation than the one with a deterministic threshold. In other words, we will deal with the following tail index estimator:

$$
\widehat{\gamma}_{1}^{(\mathbf{L B})}:=\frac{1}{n \overline{\mathbf{F}}_{n}^{(\mathbf{L B})}\left(X_{n-k: n}\right)} \sum_{i=1}^{k} \frac{\mathbf{F}_{n}^{(\mathbf{L B})}\left(X_{n-i+1: n}\right)}{C_{n}\left(X_{n-i+1: n}\right)} \log \frac{X_{n-i+1: n}}{X_{n-k: n}} .
$$

Note that $\mathbf{F}_{n}^{(\mathbf{L B})}(\infty)=1$ and write $\overline{\mathbf{F}}_{n}^{(\mathbf{L B})}\left(X_{n-k: n}\right)=\int_{X_{n-k: n}}^{\infty} d \mathbf{F}_{n}^{(\mathbf{L B})}(y)$. On the other hand, we have $C_{n}(x) d \mathbf{F}_{n}^{(\mathbf{L B})}(x)=\mathbf{F}_{n}^{(\mathbf{L B})}(x) d F_{n}(x)$ (see, e.g., Strzalkowska-Kominiak and Stute, 2009), then

$$
\overline{\mathbf{F}}_{n}^{(\mathbf{L B})}\left(X_{n-k: n}\right)=\int_{X_{n-k: n}}^{\infty} \frac{\mathbf{F}_{n}^{(\mathbf{L B})}(x)}{C_{n}(x)} d F_{n}(x)=\frac{1}{n} \sum_{i=1}^{k} \frac{\mathbf{F}_{n}^{(\mathbf{L B})}\left(X_{n-i+1: n}\right)}{C_{n}\left(X_{n-i+1: n}\right)} .
$$

This allows us to rewrite the new estimator into

$$
\widehat{\gamma}_{1}^{(\mathbf{L B})}:=\sum_{i=1}^{k} a_{n}^{(i)} \log \frac{X_{n-i+1: n}}{X_{n-k: n}}
$$


where

$$
a_{n}^{(i)}:=\frac{\mathbf{F}_{n}^{(\mathbf{L B})}\left(X_{n-i+1: n}\right)}{C_{n}\left(X_{n-i+1: n}\right)} / \sum_{i=1}^{k} \frac{\mathbf{F}_{n}^{(\mathbf{L B})}\left(X_{n-i+1: n}\right)}{C_{n}\left(X_{n-i+1: n}\right)} .
$$

It is worth mentioning that for complete data, we have $n \equiv N$ and $\mathbf{F}_{n} \equiv F_{n} \equiv C_{n}$, it follows that $a_{n}^{(i)} \equiv k^{-1}, i=1, \ldots, k$ and consequently both $\widehat{\gamma}_{1}^{(\mathbf{L B})}$ and $\widehat{\gamma}_{1}^{(\mathbf{W})}$ reduce to the classical Hill estimator (Hill, 1975). The consistency and asymptotic normality of $\widehat{\gamma}_{1}^{(\mathbf{L B})}$ will be achieved through a weak approximation of the corresponding tail Lynden-Bell process that we define by

$$
\mathbf{D}_{n}^{(\mathbf{L B})}(x):=\sqrt{k}\left(\frac{\overline{\mathbf{F}}_{n}^{(\mathbf{L B})}\left(X_{n-k: n} x\right)}{\overline{\mathbf{F}}_{n}^{(\mathbf{L B})}\left(X_{n-k: n}\right)}-x^{-1 / \gamma_{1}}\right), x>0 .
$$

The rest of the paper is organized as follows. In Section 2, we provide our main results whose proofs are postponed to Section 4 . The finite sample behavior of the proposed estimator $\widehat{\gamma}_{1}^{(\mathbf{L B})}$ is checked by simulation in Section 3, where a comparison with the one recently introduced by Benchaira et al. (2016a) is made as well.

\section{Main results}

We basically have three main results. The first one, that we give in Theorem 2.1, consists in an asymptotic relation between the above mentioned estimators of the distribution tail, namely $\overline{\mathbf{F}}_{n}^{(\mathbf{W})}$ and $\overline{\mathbf{F}}_{n}^{(\mathbf{L B})}$. This in turn is instrumental to the Gaussian approximation of the tail Lynden-Bell process $\mathbf{D}_{n}^{(\mathbf{L B})}(x)$ stated in Theorem 2.2. Finally, in Theorem 2.3, we deduce the asymptotic behavior of the tail index estimator $\widehat{\gamma}_{1}^{(\mathbf{L B})}$.

Theorem 2.1. Assume that both $\mathbf{F}$ and $\mathbf{G}$ satisfy the second-order conditions (1.2) and (1.3) respectively with $\gamma_{1}<\gamma_{2}$. Let $k=k_{n}$ be a random sequence of integers such that, given $n=m, k_{m} \rightarrow \infty$ and $k_{m} / m \rightarrow 0$, as $N \rightarrow \infty$, then, for any $x_{0}>0$, we have

$$
\sup _{x \geq x_{0}} x^{1 / \gamma_{1}} \frac{\left|\overline{\mathbf{F}}_{n}^{(\mathbf{W})}\left(X_{n-k: n} x\right)-\overline{\mathbf{F}}_{n}^{(\mathbf{L B})}\left(X_{n-k: n} x\right)\right|}{\overline{\mathbf{F}}\left(X_{n-k: n}\right)}=O_{\mathbf{P}}\left((k / n)^{\gamma_{1} / \gamma}\right) \text {. }
$$

Theorem 2.2. Assume that the assumptions of Theorem 2.1 hold and given $n=m$,

$$
k_{m}^{1+\gamma_{1} /(2 \gamma)} / m \rightarrow 0
$$

and $\sqrt{k_{m}} \mathbf{A}_{0}\left(m / k_{m}\right)=O(1)$, as $N \rightarrow \infty$. Then, for any $x_{0}>0$ and $0<\epsilon<1 / 2-\gamma / \gamma_{2}$, we have

$$
\sup _{x \geq x_{0}} x^{(1 / 2-\epsilon) / \gamma-1 / \gamma_{2}}\left|\mathbf{D}_{n}^{(\mathbf{L B})}(x)-\boldsymbol{\Gamma}(x ; \mathbf{W})-x^{-1 / \gamma_{1}} \frac{x^{\tau_{1} / \gamma_{1}}-1}{\gamma_{1} \tau_{1}} \sqrt{k} \mathbf{A}_{0}(n / k)\right|=o_{\mathbf{P}}(1) .
$$


Theorem 2.3. Assume that (1.1) holds with $\gamma_{1}<\gamma_{2}$ and let $k=k_{n}$ be a random sequence of integers such that given $n=m, k_{m} \rightarrow \infty$ and $k_{m} / m \rightarrow 0$, as $N \rightarrow \infty$, then $\widehat{\gamma}_{1}^{(\mathbf{L B})} \stackrel{\mathbf{P}}{\rightarrow} \gamma_{1}$. Assume further that the assumptions of Theorem 2.2 hold, then

$$
\begin{aligned}
\sqrt{k}\left(\widehat{\gamma}_{1}^{(\mathbf{L B})}-\gamma_{1}\right) & =\frac{\sqrt{k} \mathbf{A}_{0}(n / k)}{1-\tau_{1}}-\gamma \mathbf{W}(1) \\
& +\frac{\gamma}{\gamma_{1}+\gamma_{2}} \int_{0}^{1}\left(\gamma_{2}-\gamma_{1}-\gamma \log s\right) s^{-\gamma / \gamma_{2}-1} \mathbf{W}(s) d s+o_{\mathbf{P}}(1) .
\end{aligned}
$$

If, in addition, we suppose that, given $n=m, \sqrt{k_{m}} \mathbf{A}_{\mathbf{F}}^{*}\left(m / k_{m}\right) \rightarrow \lambda<\infty$, then

$$
\sqrt{k}\left(\widehat{\gamma}_{1}^{(\mathbf{L B})}-\gamma_{1}\right) \stackrel{\mathcal{D}}{\rightarrow} \mathcal{N}\left(\frac{\lambda}{1-\tau_{1}}, \sigma^{2}\right) \text {, as } N \rightarrow \infty \text {. }
$$

\section{Simulation study}

In this section, we illustrate the finite sample behavior of $\widehat{\gamma}_{1}^{(\mathbf{L B})}$ and, at the same time, we compare it with $\widehat{\gamma}_{1}^{(\mathbf{W})}$. To this end, we consider two sets of truncated and truncation data, both drawn from Burr's model: $\overline{\mathbf{F}}(x)=\left(1+x^{1 / \delta}\right)^{-\delta / \gamma_{1}}, \overline{\mathbf{G}}(x)=\left(1+x^{1 / \delta}\right)^{-\delta / \gamma_{2}}$, $x \geq 0$, where $\delta, \gamma_{1}, \gamma_{2}>0$. The corresponding percentage of observed data is equal to $p=\gamma_{2} /\left(\gamma_{1}+\gamma_{2}\right)$. We fix $\delta=1 / 4$ and choose the values 0.6 and 0.8 for $\gamma_{1}$ and $55 \%, 70 \%$ and $90 \%$ for $p$. For each couple $\left(\gamma_{1}, p\right)$, we solve the equation $p=\gamma_{2} /\left(\gamma_{1}+\gamma_{2}\right)$ to get the pertaining $\gamma_{2}$-value. We vary the common size $N$ of both samples $\left(\mathbf{X}_{1}, \ldots, \mathbf{X}_{N}\right)$ and $\left(\mathbf{Y}_{1}, \ldots, \mathbf{Y}_{N}\right)$, then for each size, we generate 1000 independent replicates. Our overall results are taken as the empirical means of the results obtained through all repetitions. To determine the optimal number of top statistics used in the computation of the tail index estimate values, we use the algorithm of Reiss and Thomas (2007), page 137. Our illustration and comparison are made with respect to the estimators absolute biases (abs bias) and the roots of their mean squared errors (rmse). We summarize the simulation results in Tables 3.1, 3.2 and 3.3 for $\gamma_{1}=0.6$ and in Tables 3.4, 3.5 and 3.6 for $\gamma_{1}=0.8$. After the inspection of all the tables, two conclusions can be drawn regardless of the situation. First, the estimation accuracy of both estimators decreases when the truncation percentage increases and this was quite expected. Second, we notice that the newly proposed estimator $\widehat{\gamma}_{1}^{(\mathbf{L B})}$ and $\widehat{\gamma}_{1}^{(\mathbf{W})}$ behave equally well.

\section{Proofs}

4.1. Proof Theorem 2.1. For $x \geq x_{0}$ we have

$$
\mathbf{F}_{n}^{(\mathbf{W})}\left(X_{n-k: n} x\right)=\exp \left\{-\int_{X_{n-k: n} x}^{\infty} \frac{d F_{n}(y)}{C_{n}(y)}\right\} .
$$




\begin{tabular}{rr||rrr|||ccc}
\hline \multicolumn{8}{c||}{$\gamma_{1}=0.6 ; p=0.55$} \\
\hline \multicolumn{1}{c}{$\widehat{\gamma}_{1}^{(\mathbf{L B})}$} & \multicolumn{3}{|c}{$\widehat{\gamma}_{1}^{(\mathbf{W})}$} \\
\hline \hline 100 & 54 & 0.0407 & 0.2381 & 26 & 0.0443 & 0.2328 & 26 \\
200 & 109 & 0.0378 & 0.2610 & 36 & 0.0358 & 0.2532 & 37 \\
300 & 165 & 0.0352 & 0.2359 & 36 & 0.0323 & 0.2315 & 37 \\
500 & 274 & 0.0199 & 0.2290 & 61 & 0.0185 & 0.2238 & 61 \\
1000 & 549 & 0.0074 & 0.1763 & 112 & 0.0068 & 0.1748 & 112 \\
3000 & 1649 & 0.0036 & 0.0982 & 350 & 0.0037 & 0.0981 & 352 \\
5000 & 2747 & 0.0007 & 0.1066 & 432 & 0.0007 & 0.1065 & 432 \\
\hline \hline
\end{tabular}

TABLE 3.1. Estimation results of Lynden-Bell based (leftt pannel) and Woodroofe based (right pannel) estimators of the shape parameter $\gamma_{1}=0.6$ of Burr's model through 1000 right-truncated samples with 45\%-truncation rate.

\begin{tabular}{rr||rcr|||ccc}
\multicolumn{8}{c||}{$\gamma_{1}=0.6 ; p=0.7$} \\
\hline \multicolumn{1}{c}{$\widehat{\gamma}_{1}^{(\mathbf{L B})}$} & \multicolumn{3}{c}{$\widehat{\gamma}_{1}^{(\mathbf{W})}$} \\
\hline \hline 100 & 69 & 0.0158 & 0.2451 & 25 & 0.0144 & 0.2428 & 25 \\
200 & 140 & 0.0095 & 0.1871 & 39 & 0.0089 & 0.1866 & 39 \\
300 & 210 & 0.0085 & 0.1590 & 61 & 0.0082 & 0.1587 & 61 \\
500 & 348 & 0.0074 & 0.1294 & 76 & 0.0072 & 0.1293 & 76 \\
1000 & 699 & 0.0063 & 0.1014 & 124 & 0.0062 & 0.1014 & 124 \\
3000 & 2096 & 0.0053 & 0.0962 & 246 & 0.0053 & 0.0962 & 246 \\
5000 & 3498 & 0.0036 & 0.0984 & 400 & 0.0036 & 0.0984 & 400 \\
\hline \hline
\end{tabular}

TABLE 3.2. Estimation results of Lynden-Bell based (left pannel) and Woodroofe based (right pannel) estimators of the shape parameter $\gamma_{1}=0.6$ of Burr's model through 1000 right-truncated samples with 30\%-truncation rate. 


\begin{tabular}{rr||rcr|||ccc}
\multicolumn{8}{c||}{$\gamma_{1}=0.6 ; p=0.9$} \\
\hline \multicolumn{1}{c}{$\widehat{\gamma}_{1}^{(\mathbf{L B})}$} & \multicolumn{3}{|c}{$\widehat{\gamma}_{1}^{(\mathbf{W})}$} \\
\hline \hline 100 & 90 & 0.0073 & 0.1779 & 21 & 0.0070 & 0.1778 & 21 \\
200 & 180 & 0.0066 & 0.1208 & 54 & 0.0064 & 0.1208 & 54 \\
300 & 270 & 0.0055 & 0.1133 & 88 & 0.0056 & 0.1133 & 88 \\
500 & 450 & 0.0050 & 0.0864 & 125 & 0.0050 & 0.0863 & 125 \\
1000 & 898 & 0.0030 & 0.0614 & 189 & 0.0029 & 0.0614 & 189 \\
3000 & 2702 & 0.0016 & 0.0494 & 398 & 0.0016 & 0.0494 & 398 \\
5000 & 4496 & 0.0010 & 0.0112 & 467 & 0.0010 & 0.0112 & 467 \\
\hline \hline
\end{tabular}

TABLE 3.3. Estimation results of Lynden-Bell based (left pannel) and Woodroofe based (right pannel) estimators of the shape parameter $\gamma_{1}=0.6$ of Burr's model through 1000 right-truncated samples with 10\%-truncation rate.

\begin{tabular}{rr||rcc||ccc}
\hline \multicolumn{8}{c}{$\gamma_{1}=0.8 ; p=0.55$} \\
\hline \multicolumn{1}{c}{$\widehat{\gamma}_{1}^{(\mathbf{L B})}$} & \multicolumn{1}{c||}{$\widehat{\gamma}_{1}^{(\mathbf{W})}$} \\
\hline \hline 100 & 55 & 0.0570 & 0.3330 & 30 & 0.0636 & 0.3167 & 31 \\
200 & 110 & 0.0401 & 0.3604 & 33 & 0.0347 & 0.3453 & 35 \\
300 & 164 & 0.0252 & 0.2563 & 69 & 0.0272 & 0.2530 & 71 \\
500 & 276 & 0.0227 & 0.1807 & 112 & 0.0216 & 0.1794 & 113 \\
1000 & 551 & 0.0148 & 0.1795 & 196 & 0.0142 & 0.1788 & 197 \\
3000 & 1647 & 0.0124 & 0.1794 & 525 & 0.0121 & 0.1783 & 525 \\
5000 & 2751 & 0.0075 & 0.1260 & 688 & 0.0074 & 0.1259 & 688 \\
\hline \hline
\end{tabular}

TABLE 3.4. Estimation results of Lynden-Bell based (left pannel) and Woodroofe based (right pannel) estimators of the shape parameter $\gamma_{1}=0.8$ of Burr's model through 1000 right-truncated samples with 45\%-truncation rate. 


\begin{tabular}{rr||rrr|||ccc}
\multicolumn{8}{c||}{$\gamma_{1}=0.8 ; p=0.7$} \\
\hline \multicolumn{1}{c}{$\widehat{\gamma}_{1}^{(\mathbf{L B})}$} & \multicolumn{3}{|c}{$\widehat{\gamma}_{1}^{(\mathbf{W})}$} \\
\hline \hline 100 & 69 & 0.0217 & 0.3827 & 28 & 0.0195 & 0.3787 & 28 \\
200 & 139 & 0.0203 & 0.2918 & 59 & 0.0194 & 0.2905 & 59 \\
300 & 210 & 0.0189 & 0.1857 & 66 & 0.0184 & 0.1852 & 66 \\
500 & 348 & 0.0143 & 0.1593 & 113 & 0.0140 & 0.1591 & 113 \\
1000 & 700 & 0.0049 & 0.1205 & 230 & 0.0049 & 0.1204 & 230 \\
3000 & 2100 & 0.0037 & 0.0886 & 449 & 0.0038 & 0.0886 & 449 \\
5000 & 3500 & 0.0031 & 0.0857 & 500 & 0.0031 & 0.0857 & 500 \\
\hline \hline
\end{tabular}

TABLE 3.5. Estimation results of Lynden-Bell based (left pannel) and Woodroofe based (right pannel) estimators of the shape parameter $\gamma_{1}=0.8$ of Burr's model through 1000 right-truncated samples with 30\%-truncation rate.

\begin{tabular}{rr||rrr|||ccc}
\hline \multicolumn{8}{c||}{$\gamma_{1}=0.8 ; p=0.9$} \\
\hline \multicolumn{1}{c}{$\widehat{\gamma}_{1}^{(\mathbf{L B})}$} & \multicolumn{3}{|c}{$\widehat{\gamma}_{1}^{(\mathbf{W})}$} \\
\hline \hline 100 & 89 & 0.0380 & 0.1833 & 38 & 0.0369 & 0.1827 & 38 \\
200 & 179 & 0.0345 & 0.1383 & 80 & 0.0342 & 0.1383 & 80 \\
300 & 269 & 0.0173 & 0.1014 & 99 & 0.0175 & 0.1013 & 99 \\
500 & 450 & 0.0108 & 0.0927 & 143 & 0.0106 & 0.0926 & 143 \\
1000 & 899 & 0.0021 & 0.0729 & 260 & 0.0021 & 0.0729 & 260 \\
3000 & 2697 & 0.0013 & 0.0591 & 443 & 0.0013 & 0.0591 & 443 \\
5000 & 4500 & 0.0001 & 0.0309 & 997 & 0.0001 & 0.0309 & 997 \\
\hline \hline
\end{tabular}

TABLE 3.6. Estimation results of Lynden-Bell based (left pannel) and Woodroofe based (right pannel) estimators of the shape parameter $\gamma_{1}=0.8$ of Burr's model through 1000 right-truncated samples with 10\%-truncation rate. 
We show that the latter exponent is negligible in probability uniformly over $x \geq x_{0}$. Indeed, note that both $F_{n}(y) / F(y)$ and $C(y) / C_{n}(y)$ are stochastically bounded from above on $y<$ $X_{n: n}$ (see, e.g., Shorack and Wellner, 1986, page 415 and Strzalkowska-Kominiak and Stute, 2009 , respectively), it follows that

$$
-\int_{X_{n-k: n} x}^{\infty} \frac{d F_{n}(y)}{C_{n}(y)}=O_{\mathbf{P}}(1) \int_{X_{n-k: n} x}^{\infty} \frac{d F(y)}{C(y)} .
$$

By a change of variables we have

$$
\int_{X_{n-k: n} x}^{\infty} \frac{d \bar{F}(y)}{C(y)}=\frac{\bar{F}\left(X_{n-k: n}\right)}{C\left(X_{n-k: n}\right)}\left(\int_{x}^{\infty} \frac{C\left(X_{n-k: n}\right)}{C\left(X_{n-k: n} t\right)} d \frac{\bar{F}\left(X_{n-k: n} t\right)}{\bar{F}\left(X_{n-k: n}\right)}\right) .
$$

Recall that $X_{n-k: n} \stackrel{\mathbf{P}}{\rightarrow} \infty$ and that $\bar{F}$ is regularly varying at infinity with index $-1 / \gamma$. On the other hand, from Assertion ( $i$ ) of Lemma A.2 in Benchaira et al. (2016a) we deduce that 1/C is also regularly varying at infinity with index $1 / \gamma_{2}$. Thus, we may apply Potters inequalities, see e.g. Proposition B.1.10 in de Haan and Ferreira (2006), to both $\bar{F}$ and 1/C to write: for all large $N$, any $t \geq x_{0}$ and any sufficiently small $\delta, \nu>0$, with large probability,

$$
\left|\frac{\bar{F}\left(X_{n-k: n} t\right)}{\bar{F}\left(X_{n-k: n}\right)}-t^{-1 / \gamma}\right|<\delta t^{-1 / \gamma \pm \nu} \text { and }\left|\frac{C\left(X_{n-k: n}\right)}{C\left(X_{n-k: n} t\right)}-t^{1 / \gamma_{2}}\right|<\delta t^{1 / \gamma_{2} \pm \nu},
$$

where $t^{ \pm a}:=\max \left(t^{a}, t^{-a}\right)$. These two inequalities may be rewritten, into

$$
\frac{\bar{F}\left(X_{n-k: n} t\right)}{\bar{F}\left(X_{n-k: n}\right)}=t^{-1 / \gamma}\left(1+o_{\mathbf{P}}\left(t^{ \pm \nu}\right)\right) \text { and } \frac{C\left(X_{n-k: n}\right)}{C\left(X_{n-k: n} t\right)}=t^{1 / \gamma_{2}}\left(1+o_{\mathbf{P}}\left(t^{ \pm \nu}\right)\right)
$$

uniformly on $t \geq x_{0}$. This leads to

$$
\int_{x}^{\infty} \frac{C\left(X_{n-k: n}\right)}{C\left(X_{n-k: n} t\right)} d \frac{\bar{F}\left(X_{n-k: n} t\right)}{\bar{F}\left(X_{n-k: n}\right)}=-\frac{\gamma_{1}}{\gamma} x^{-1 / \gamma_{1}}\left(1+o_{\mathbf{P}}\left(x^{ \pm \nu}\right)\right) .
$$

In view of (1.4), Benchaira et al. (2016a) showed, in Lemma A1, that $\bar{F}(y)=(1+o(1)) c_{1} y^{-1 / \gamma}$ and $\bar{G}(y)=(1+o(1)) c_{2} y^{-1 / \gamma_{2}}$ as $y \rightarrow \infty$, for some constants $c_{1}, c_{2}>0$. In other words, $\mathbb{U}_{F}(s)=(1+o(1))\left(c_{1} s\right)^{\gamma}$ as $s \rightarrow \infty$, and $C(y)=(1+o(1)) c_{2} y^{-1 / \gamma_{2}}$ as $y \rightarrow \infty$. On the other hand, from Lemma A4 in Benchaira et al. (2016a), we have $X_{n-k: n}=\left(1+o_{\mathbf{P}}(1)\right) \mathbb{U}_{F}(n / k)$, it follows that $X_{n-k: n}=\left(1+o_{\mathbf{P}}(1)\right) c_{1}^{\gamma}(k / n)^{-\gamma}$. Note that $1-\gamma / \gamma_{2}=\gamma / \gamma_{1}$, hence

$$
\frac{\bar{F}\left(X_{n-k: n}\right)}{C\left(X_{n-k: n}\right)}=\left(1+o_{\mathbf{P}}(1)\right) c_{1}^{\gamma / \gamma_{2}} c_{2}^{-1}(k / n)^{\gamma / \gamma_{1}} \text {. }
$$

Plugging results (4.10) and (4.11) in equation (4.8) yields

$$
\int_{X_{n-k: n} x}^{\infty} \frac{d \bar{F}(y)}{C(y)}=(k / n)^{\gamma / \gamma_{1}} c_{1}^{\gamma / \gamma_{2}} c_{2}^{-1} \gamma_{1} x^{-1 / \gamma_{1}}\left(1+o_{\mathbf{P}}\left(x^{ \pm \nu}\right)\right) .
$$


By combining equations (4.7) and (4.12), we obtain

$$
\int_{X_{n-k: n} x}^{\infty} \frac{d F_{n}(y)}{C_{n}(y)}=O_{\mathbf{P}}(1)(k / n)^{\gamma / \gamma_{1}} x^{-1 / \gamma_{1}}\left(1+o_{\mathbf{P}}\left(x^{ \pm \nu}\right)\right),
$$

which obviously tends to zero in probability (uniformly on $x \geq x_{0}$ ). We may now apply Taylor's expansion $e^{t}=1+t+O\left(t^{2}\right)$, as $t \rightarrow 0$, to get

$$
\exp \left\{-\int_{X_{n-k: n} x}^{\infty} \frac{d F_{n}(y)}{C_{n}(y)}\right\}=1-\int_{X_{n-k: n} x}^{\infty} \frac{d F_{n}(y)}{C_{n}(y)}+O_{\mathbf{P}}\left(\int_{X_{n-k: n} x}^{\infty} \frac{d F_{n}(y)}{C_{n}(y)}\right)^{2}, N \rightarrow \infty .
$$

In other words, we have

$$
\overline{\mathbf{F}}_{n}^{(\mathbf{W})}\left(X_{n-k: n} x\right)=\int_{X_{n-k: n} x}^{\infty} \frac{d F_{n}(y)}{C_{n}(y)}+R_{n 1}(x), N \rightarrow \infty,
$$

where $R_{n 1}(x):=O_{\mathbf{P}}\left((k / n)^{2 \gamma / \gamma_{1}}\right) x^{-2 / \gamma_{1}}\left(1+o_{\mathbf{P}}\left(x^{ \pm \nu}\right)\right)$. Next, we show that

$$
\overline{\mathbf{F}}_{n}^{(\mathbf{L B})}\left(X_{n-k: n} x\right)=\int_{X_{n-k: n} x}^{\infty} \frac{d F_{n}(y)}{C_{n}(y)}+R_{n 2}(x), N \rightarrow \infty .
$$

Observe that, by taking the logarithme then its exponential in the definition of $\mathbf{F}_{n}^{(\mathbf{L B})}(x)$, we have

$$
\mathbf{F}_{n}^{(\mathbf{L B})}\left(X_{n-k: n} x\right)=\exp \left\{\sum_{i=1}^{n} \mathbf{1}\left(X_{i: n}>X_{n-k: n} x\right) \log \left(1-\frac{1}{n C_{n}\left(X_{i: n}\right)}\right)\right\}
$$

which may be rewritten into $\exp \left\{n \int_{x}^{\infty} \log \left(1-\frac{1}{n C_{n}\left(X_{n-k: n} y\right)}\right) d F_{n}\left(X_{n-k: n} y\right)\right\}$. To get approximation (4.15) it suffices to apply successively, in the previous quantity, Taylor's expansions $e^{t}=1+t+O\left(t^{2}\right)$ and $\log (1-t)=-t+O\left(t^{2}\right)($ as $t \rightarrow 0)$ with similar arguments as above (we omit further details). Combining (4.14) and (4.15) and setting $R_{n}(x):=$ $R_{n 1}(x)-R_{n 2}(x)$ yield

$$
\overline{\mathbf{F}}_{n}^{(\mathbf{W})}\left(X_{n-k: n} x\right)-\overline{\mathbf{F}}_{n}^{(\mathbf{L B})}\left(X_{n-k: n} x\right)=R_{n}(x), N \rightarrow \infty .
$$

On the other hand, by once again using Taylor's expansion, we write

$$
\overline{\mathbf{F}}\left(X_{n-k: n}\right)=\int_{X_{n-k: n}}^{\infty} \frac{d F(y)}{C(y)}+\widetilde{R}_{n}(x), N \rightarrow \infty .
$$

From equation (4.12), we infer that $\overline{\mathbf{F}}\left(X_{n-k: n}\right)=c_{2}^{-1} c_{1}^{1-\gamma / \gamma_{1}}(k / n)^{\gamma / \gamma_{1}}\left(1+o_{\mathbf{P}}(1)\right)$, which implies, in view of (4.16), that

$$
x^{1 / \gamma_{1}} \frac{\overline{\mathbf{F}}_{n}^{(\mathbf{L B})}\left(X_{n-k: n} x\right)-\overline{\mathbf{F}}_{n}^{(\mathbf{W})}\left(X_{n-k: n} x\right)}{\overline{\mathbf{F}}\left(X_{n-k: n}\right)}=O_{\mathbf{P}}\left((k / n)^{\gamma / \gamma_{1}}\right) x^{-1 / \gamma_{1} \pm \nu} .
$$


Observe now that, for a sufficiently small $\nu>0$, we have $x^{-1 / \gamma_{1} \pm \nu}=O_{\mathbf{P}}(1)$, uniformly on $x \geq x_{0}>0$, as sought.

4.2. Proof Theorem 2.2. In a similar way to what is done with $\mathbf{D}_{n}^{(\mathbf{W})}(x)$, in the proof of Theorem 2.1 in Benchaira et al. (2016a), we decompose $k^{-1 / 2} \mathbf{D}_{n}^{(\mathbf{L B})}(x)$ into the sum of

$$
\begin{gathered}
\mathbf{N}_{n 1}(x):=x^{-1 / \gamma_{1}} \frac{\overline{\mathbf{F}}_{n}^{(\mathbf{L B})}\left(X_{n-k: n} x\right)-\overline{\mathbf{F}}\left(X_{n-k: n}\right)}{\overline{\mathbf{F}}\left(X_{n-k: n}\right)}, \\
\mathbf{N}_{n 2}(x):=-\frac{\overline{\mathbf{F}}\left(X_{n-k: n} x\right)}{\overline{\mathbf{F}}_{n}^{(\mathbf{L B})}\left(X_{n-k: n}\right)} \frac{\overline{\mathbf{F}}_{n}^{(\mathbf{L B})}\left(X_{n-k: n}\right)-\overline{\mathbf{F}}\left(X_{n-k: n}\right)}{\overline{\mathbf{F}}\left(X_{n-k: n}\right)}, \\
\mathbf{N}_{n 3}(x):=\left(\frac{\overline{\mathbf{F}}\left(X_{n-k: n} x\right)}{\overline{\mathbf{F}}_{n}^{(\mathbf{L B})}\left(X_{n-k: n}\right)}-x^{-1 / \gamma_{1}}\right) \frac{\overline{\mathbf{F}}_{n}^{(\mathbf{L B})}\left(X_{n-k: n} x\right)-\overline{\mathbf{F}}\left(x X_{n-k: n}\right)}{\overline{\mathbf{F}}\left(X_{n-k: n} x\right)},
\end{gathered}
$$

and $\mathbf{N}_{n 4}(x):=\overline{\mathbf{F}}\left(X_{n-k: n} x\right) / \overline{\mathbf{F}}\left(X_{n-k: n}\right)-x^{-1 / \gamma_{1}}$. If we let

$$
\mathbf{M}_{n 1}(x):=x^{-1 / \gamma_{1}} \frac{\overline{\mathbf{F}}_{n}^{(\mathbf{W})}\left(X_{n-k: n} x\right)-\overline{\mathbf{F}}\left(X_{n-k: n}\right)}{\overline{\mathbf{F}}\left(X_{n-k: n}\right)},
$$

then, by applying Theorem 2.1, we have $x^{1 / \gamma_{1}} \mathbf{N}_{n 1}(x)=x^{1 / \gamma_{1}} \mathbf{M}_{n 1}(x)+x^{-1 / \gamma_{1}} O_{\mathbf{P}}\left((k / n)^{\gamma / \gamma_{1}}\right)$, uniformly on $x \geq x_{0}$. By assumption we have $k^{1+\gamma_{1} /(2 \gamma)} / n \stackrel{\mathbf{P}}{\rightarrow} 0$, which is equivalent to $\sqrt{k}(k / n)^{\gamma / \gamma_{1}} \stackrel{\mathbf{P}}{\rightarrow} 0$ as $N \rightarrow \infty$, therefore

$$
x^{1 / \gamma_{1}} \sqrt{k} \mathbf{N}_{n 1}(x)=x^{1 / \gamma_{1}} \sqrt{k} \mathbf{M}_{n 1}(x)+o_{\mathbf{P}}\left(x^{-1 / \gamma_{1}}\right) .
$$

In view of this representation we show that, both $\mathbf{D}_{n}^{(\mathbf{W})}(x)$ and $\mathbf{D}_{n}^{(\mathbf{L B})}(x)$ are (weakly) approximated, in the probability space $(\Omega, \mathcal{A}, \mathbf{P})$, by the same Gaussian process $\boldsymbol{\Gamma}(x ; \mathbf{W})$ given in (1.5). Indeed, for a sufficiently small $\epsilon>0$, and $0<\eta<1 / 2$, Benchaira et al. (2016a) (see the beginning of the proof of Theorem 2.1 therein), showed that

$$
x^{1 / \gamma_{1}} \sqrt{k} \mathbf{M}_{n 1}(x)=\Phi(x)+o_{\mathbf{P}}\left(x^{(1-\eta) / \gamma \pm \epsilon}\right),
$$

where $\Phi(x):=x^{1 / \gamma}\left\{\frac{\gamma}{\gamma_{1}} \mathbf{W}\left(x^{-1 / \gamma}\right)+\frac{\gamma}{\gamma_{1}+\gamma_{2}} \int_{0}^{1} t^{-\gamma / \gamma_{2}-1} \mathbf{W}\left(x^{-1 / \gamma} t\right) d t\right\}$. Then by using representation (4.17), we get $x^{1 / \gamma_{1}} \sqrt{k} \mathbf{N}_{n 1}(x)=\Phi(x)+o_{\mathbf{P}}\left(x^{-1 / \gamma_{1}}\right)+o_{\mathbf{P}}\left(x^{(1-\eta) / \gamma \pm \epsilon}\right)$. In particular for $x=1$, we have

$$
\sqrt{k}\left(\frac{\overline{\mathbf{F}}_{n}^{(\mathbf{L B})}\left(X_{n-k: n}\right)}{\overline{\mathbf{F}}\left(X_{n-k: n}\right)}-1\right)=\sqrt{k} \mathbf{N}_{n 1}(1)=\Phi(1)+o_{\mathbf{P}}(1),
$$


leading to $\overline{\mathbf{F}}_{n}^{(\mathbf{L B})}\left(X_{n-k: n}\right) / \overline{\mathbf{F}}\left(X_{n-k: n}\right) \stackrel{\mathbf{P}}{\rightarrow} 1$, as $N \rightarrow \infty$. By applying Potters inequalities to $\overline{\mathbf{F}}$ (as it was done for $\bar{F}$ in (4.11)) together with the previous limit, we obtain

$$
\frac{\overline{\mathbf{F}}\left(X_{n-k: n} x\right)}{\overline{\mathbf{F}}_{n}^{(\mathbf{L B})}\left(X_{n-k: n}\right)}=\left(1+O_{\mathbf{P}}\left(x^{ \pm \epsilon}\right)\right) x^{-1 / \gamma_{1}} .
$$

By combining (4.18) and(4.19), we get $x^{1 / \gamma_{1}} \sqrt{k} \mathbf{N}_{n 2}(x)=-\Phi(1)+o_{\mathbf{P}}\left(x^{ \pm \epsilon}\right)$. For the third term $\mathbf{N}_{n 3}(x)$, we use similar arguments to show that

$$
x^{1 / \gamma_{1}} \sqrt{k} \mathbf{N}_{n 3}(x)=o_{\mathbf{P}}\left(x^{-1 / \gamma_{1} \pm \epsilon}\right)+o_{\mathbf{P}}\left(x^{-1 / \gamma_{1}+(1-\eta) / \gamma \pm \epsilon}\right) .
$$

Observe that $x^{1 / \gamma_{1}-\left(1-\eta_{0}\right) / \gamma_{O}}\left(x^{-1 / \gamma_{1} \pm \epsilon}\right)$ and $x^{1 / \gamma_{1}-\left(1-\eta_{0}\right) / \gamma_{O}}\left(x^{-1 / \gamma_{1}+(1-\eta) / \gamma \pm \epsilon}\right)$ respectively equal $o_{\mathbf{P}}\left(x^{-\left(1-\eta_{0}\right) / \gamma \pm \epsilon}\right)$ and $o_{\mathbf{P}}\left(x^{\left(\eta-\eta_{0}\right) / \gamma \pm \epsilon}\right)$, for $\gamma / \gamma_{2}<\eta_{0}<\eta<1 / 2$, and that both the last two quantities are equal to $o_{\mathbf{P}}(1)$ for any small $\epsilon>0$ and $x \geq x_{0}>0$. Finally, by following the same steps at the end of the proof of Theorem 2.1 in Benchaira et al. (2016a), we get

$$
\sqrt{k} \mathbf{N}_{n 4}(x)=x^{-1 / \gamma_{1}} \frac{x^{\tau_{1} / \gamma_{1}}-1}{\gamma_{1} \tau_{1}} \sqrt{k} \mathbf{A}_{0}(n / k)+o_{\mathbf{p}}\left(x^{-1 / \gamma_{1}+(1-\eta) / \gamma \pm \epsilon}\right) .
$$

Consequently, we have

$$
x^{1 / \gamma_{1}-\left(1-\eta_{0}\right) / \gamma}\left\{\mathbf{D}_{n}^{(\mathbf{L B})}(x)-\boldsymbol{\Gamma}(x ; \mathbf{W})-x^{-1 / \gamma_{1}} \frac{x^{\tau_{1} / \gamma_{1}}-1}{\gamma_{1} \tau_{1}} \sqrt{k} \mathbf{A}_{0}(n / k)\right\}=o_{\mathbf{P}}(1),
$$

uniformly over $x \geq x_{0}$. Recall that $1 / \gamma_{1}=1 / \gamma-1 / \gamma_{2}$, then letting $\eta_{0}:=1 / 2-\xi$ yields $0<\xi<1 / 2-\gamma / \gamma_{2}$ and achieves the proof.

4.3. Proof of Theorem 2.3. The proof is similar, mutatis mutandis, as that of Corollary 3.1 in Benchaira et al. (2016a). Therefore we omit the details.

\section{Concluding note}

On the basis of Lynden-Bell integration, we introduced a new estimator for the tail index of right-truncated heavy-tailed data by considering a random threshold. This estimator may be an alternative to that, based on Woodroofe integration, recently proposed by Benchaira et al. (2016a). Indeed, the simulation results show that there is an equivalence between the asymptotic behaviors of both estimators with respect to biases and rmse's. However, from a theoritical point of view, the asymptotic normality of the former requires an additional condition on the sample fraction $k$ of upper order statistics, namely (2.6), which is stonger than the usual assumption in the context of extremes $(k / n \rightarrow 0)$. 


\section{REFERENCES}

Benchaira, S., Meraghni, D., Necir, A., 2015. On the asymptotic normality of the extreme value index for right-truncated data. Statist. Probab. Lett. 107, 378-384.

Benchaira, S., Meraghni, D., Necir, A., 2016a. Tail product-limit process for truncated data with application to extreme value index estimation. Extremes 19, 219-251.

Benchaira, S., Meraghni, D., Necir, A., 2016b. Kernel estimation of the tail index of a right-truncated Pareto-type distribution. Statist. Probab. Lett. 119, 186-193.

Gardes, L., Stupfler, G., 2015. Estimating extreme quantiles under random truncation. TEST 24, 207-227.

de Haan, L., Ferreira, A., 2006. Extreme Value Theory: An Introduction. Springer.

Hill, B.M., 1975. A simple general approach to inference about the tail of a distribution. Ann. Statist. 3, 1163-1174.

Hua, L., Joe, H., 2011. Second order regular variation and conditional tail expectation of multiple risks. Insurance Math. Econom. 49, 537-546.

Lynden-Bell, D., 1971. A method of allowing for known observational selection in small samples applied to 3CR quasars. Monthly Notices Roy. Astronom. Soc. 155, 95-118.

Reiss, R.D., Thomas, M., 2007. Statistical Analysis of Extreme Values with Applications to Insurance, Finance, Hydrology and Other Fields, 3rd ed. Birkhäuser.

Resnick, S., 2006. Heavy-Tail Phenomena: Probabilistic and Statistical Modeling. Springer.

Shorack, G.R., Wellner, J. A., 1986. Empirical processes with applications to statistics. New York: John Wiley 6 Sons.

Strzalkowska-Kominiak, E., Stute, W., 2009. Martingale representations of the Lynden-Bell estimator with applications. Statist. Probab. Lett. 79, 814-820.

Woodroofe, M., 1985. Estimating a distribution function with truncated data. Ann. Statist. 13, $163-177$.

Worms, J., Worms, R., 2016. A Lynden-Bell integral estimator for extremes of randomly truncated data. Statist. Probab. Lett. 109, 106-117. 